\title{
Probability analysis of wave run-ups and air gap response of a deepwater semi-submersible platform using LH-moments estimation method
}

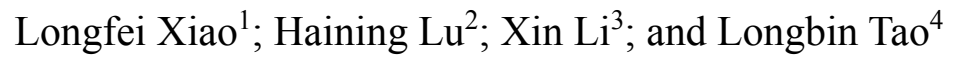

\begin{abstract}
The air gap response in harsh environments, a critical design issue for offshore platforms, is related to the wave run-ups due to wave-platform interactions and potentially results in serious wave impacts. Therefore, the reliable prediction of wave run-ups and air gap response in harsh environments is a challenging task and needs further study. In this study, probability analysis of the wave run-up data from an experimental study of a deepwater semi-submersible platform is conducted based on the three-parameter Weibull distribution model using the LH-moments method for parameter estimation. One of the highlights in the present study is that the explicit relationships between the first three LH-moments at arbitrary levels and the parameters of the Weibull distribution are established analytically. The accuracy of LH-kurtosis estimation is proposed to determine the appropriate level for probability analysis. The air gap response is more serious in quartering and beam seas than in head seas. In front of the columns along the wave incoming direction, especially the aft one, the wave run-ups show higher probability distributions leading to higher likeliness of suffering from negative air gap and wave impact accidents than the other platform areas. At the platform center, the wave run-up is significantly lower than the incident wave. The probability distributions based on LH-moments at the appropriate level can well represent large wave run-ups, except for that beyond the still-water air gap, where both measurement methods and probability analyses warrant further research.
\end{abstract}

Author keywords: Wave run-up; Air gap; Weibull distribution; Parameter estimation; LH-moments.

\footnotetext{
${ }^{1}$ Professor, State Key Laboratory of Ocean Engineering, Shanghai Jiao Tong University, 800 Dongchuan Road, Shanghai 200240, China (corresponding author). E-mail: xiaolf@sjtu.edu.cn

2 Lecturer, State Key Laboratory of Ocean Engineering, Shanghai Jiao Tong University, 800 Dongchuan Road, Shanghai 200240, China. E-mail: haining@sjtu.edu.cn

3 Associate Professor, State Key Laboratory of Ocean Engineering, Shanghai Jiao Tong University, 800
} 
Dongchuan Road, Shanghai 200240, China. E-mail: lixin@sjtu.edu.cn

${ }^{4}$ Professor, School of Marine Science and Technology, Newcastle University, Armstrong Building, Newcastle upon Tyne NE1 7RU, United Kingdom. E-mail: longbin.tao@newcastle.ac.uk

\section{Introduction}

The air gap response in harsh environments is one of the critical design issues for large-volume offshore structures, such as a semi-submersible platform, tension leg platform, and spar platform, that are widely used in offshore oil and gas production activities. The air gap is defined as the vertical distance between the underside of the lower deck and the wave run-up at the exterior of the exposed hull. Wave diffraction and run-up due to wave-hull interactions can significantly amplify incident waves and thus cause much higher wave crests under some areas of the lower decks, resulting in negative air gap response and serious wave impact on the structure (Nielsen 2003). The main complexities involved in air gap prediction include the platform motions, nonlinear wave-hull interactions, and wave run-ups along columns. Wave run-ups are always accompanied by highly nonlinear phenomena such as wave deformation, rolling, spray, and impact, making it difficult to reliably predict the air gap using current theories and methodologies. During 2004 and 2005, hurricanes Ivan, Katrina, and Rita in the Gulf of Mexico caused substantial damage to more than 100 platforms; much of this damage was caused to lower decks by serious wave run-up and wave impact (Forristall 2007). Many numerical and experimental studies have already reported on the wave run-up and air gap issues of offshore structures. Nonetheless, the prediction of the same in harsh environments remains challenging and requires more extensive studies.

Among various numerical schemes, the boundary element method (BEM) based on wave diffraction theory is commonly applied to calculate the wave run-ups along platform columns and the air gap distributions under the lower deck of large-volume floating platforms. The standard prediction for a semi-submersible platform based on linear theory showed good agreement with measurements in model tests for low wave-steepness but seriously underestimated wave run-ups in column surroundings for steep waves (Winterstein and Sweetman 2001; Simos et al. 2006). Extending the linear method to the second order clearly improved the prediction for steep waves compared to the measured data; however, wave amplifications near columns could still not be predicted exactly (Winterstein and Sweetman 2001; Stansberg et al. 2005). To deal with highly nonlinear phenomena such as wave splashes or wave breaking along with wave run-up near columns in extreme waves, a CFD simulation 
tool based on the Navier-Stokes equations was developed and the fully free-surface dynamics were described using the volume of fluid (VOF) method (Kleefsman et al. 2005; Veldman et al. 2011; Matsumoto et al. 2013). The time-consuming simulations showed good agreement with the experimental results even for the steepest wave cases in design conditions.

Experimental studies of wave run-up and air gap responses remain as the most reliable methods and are often used to verify the numerical simulations, investigate the fluid mechanism, or check the seakeeping behavior of a final design. Niedzwecki and Duggal (1992) performed small-scale model tests of wave run-ups on a circular column and demonstrated that linear diffraction theory underpredicted the wave run-ups except in the case of very low wave steepness. Martin et al. (2001) experimentally studied wave run-ups on slender columns of a semi-submersible platform in steep waves to compare the run-up predictions from several theories and found that all theories underpredicted run-up significantly. Simos et al. (2008) drew similar conclusions through small-scale model tests of the air-gap response in a floating semi-submersible platform. Shan et al. (2011) investigated the wave run-up characteristics along square columns and revealed the relationships between air gap distributions and incident wave parameters through a wide range of monochromatic wave tests of a semi-submersible platform. Matsumoto et al. (2013) reported significant run-up effects on square-section columns of a large-volume semi-submersible platform for the steepest wave tests in design conditions and evaluated the numerical predictions by using regular wave test results for different steepness values.

These studies mainly focused on the response to monochromatic waves. Relatively fewer studies have addressed the characteristics in random seastates, which are more complex in nature and certainly more important in practice (Izadparast and Niedzwecki 2009; Priyanto et al. 2014). To interpret wave run-up data with a random nature from irregular wave experiments, extreme statistics and the exceedance probability distribution need be evaluated quantitatively to determine the expected minimum air gap for design considerations. To describe nonlinear wave run-up distributions, Kriebel and Dawson (1991) proposed a two-parameter Rayleigh-Stokes model incorporating the classical Rayleigh distribution with an amplification factor based on assumptions that the first- and second-order wave run-ups are phase-locked and that the maxima occur simultaneously. Al-Humoud et al. (2002) examined the model for ocean surface waves and reported that the accuracy of the qualitative prediction was questionable. Niedzwecki et al. (2000) introduced the two-parameter Weibull 
distribution to model the wave run-ups of two compliant platforms in random seastates using model test data and revealed that the wave run-ups on circular columns could be modeled reasonably. Inderbo and Niedzwecki (2004) later reported further applications to wave run-ups on both a single rectangular column and an array of similar vertical columns. Izadparast and Niedzwecki $(2009,2010)$ developed a three-parameter Weibull-Stokes distribution model that combined theoretical information, quadratic transformation of the linear random variable, and empirical parameter estimates obtained using the L-moments method. The results showed the robustness of using L-moments for estimating the model parameters. However, it was noted that additional model flexibility might improve the accuracy in modeling the tail of some sample probability distributions.

As a generalized distribution widely used in reliability and lifetime studies (Nagatsuka et al. 2013), the three-parameter Weibull distribution was recommended and confirmed as a good model for the probability analysis of extreme waves and wave overtopping without the necessity of data censoring (Mathiesen et al. 1994; Franco and Franco 1999). On the other hand, L-moments for parameter estimation developed by Hosking (1990) represent the expectations of certain linear combinations of the overall order statistics on the whole sample data. Instead of the overall statistics, however, the tail of the probability distribution for large extremes is of much more interest, and considering less-meaningful small values by the L-moments method tends to reveal evident discrepancies (Bobee and Rasmussen 1995). Thus, the LH-moments estimation method (LHME) was recently proposed as a generalization of the L-moments method based on linear combinations of higher-order statistics to characterize the upper part of distributions (Wang 1997). This method provides an analytical means for fitting a distribution to the tail sample events without explicit sample censoring. The LHME has subsequently been used in studies for parameter estimations of various probability distributions, such as the generalized extreme value (GEV) distribution (Hewa et al. 2007), generalized logistic distribution (GLD), generalized Pareto distribution (GPD) (Meshgi and Khalili 2009), and 4-parameter kappa distribution (K4D) (Murshed et al. 2014). These applications verify the feasibility of the LH-moments method for estimating heavy-tail conditions and indicate that this method is useful for many practical applications even though the L-moments method fails to provide a feasible solution (Murshed et al. 2014).

To estimate large extreme values with small probabilities of exceedance such as large wave crests and wave run-ups in coastal and offshore engineering, the LH-moments method tends to be more 
appropriate than the L-moments method for parameter estimation of the probability distributions. Owing to the non-linearity of wave run-ups, the Weibull distribution fitting to entire sample data tends to be questionable for the extreme samples because of the effect of the smaller observations. As an alternative solution for dealing with the issue, the LH-moments method is introduced in the present study aiming to take advantage of LH-moments for characterizing the upper part of distributions and larger events in data. The LH-moments method is applied for the parameter estimation of the three-parameter Weibull distribution model in the probability analyses of the wave run-up measurements from a model test study of a deepwater semi-submersible platform. The survival conditions with 100-y return environment and three wave headings are considered. The expressions of LH-moments and explicit relationships between the first three LH-moments at arbitrary levels and the three parameters of the Weibull distribution are established analytically. The accuracy of the LH-kurtosis estimation is proposed to determine the appropriate level for probability analysis. The results of the goodness-of-fit evaluations and the estimated probability distributions are presented and discussed for different levels, including the zero level representing the L-moments. Moreover, the estimated probability distributions as well as the measured data of the wave run-ups at different platform locations are compared for head, quartering, and beam sea conditions. The exceedance probabilities of the still-water air gap and the three-hour estimates of extreme air gaps are derived based on the estimated Weibull distributions.

\section{LH-moments Estimation Method for Weibull Distribution}

\section{LH-moments Theory}

Let $X$ be a real-valued random variable with a quantile function $X(F)$. The order statistics of $X$ for a sample of size $n$ are denoted by the ascending order $X_{(1):(n)} \leq X_{(2):(n)} \leq \cdots \leq X_{(j):(n)} \leq \cdots \leq X_{(r):(n)}$. The first four LH-moments at any level of $\eta=0,1,2, \ldots$ are specified by Wang (1997) as

$$
\begin{aligned}
& \lambda_{1}^{\eta}=E\left[X_{(\eta+1):(\eta+1)}\right] \\
& \lambda_{2}^{\eta}=\frac{1}{2} E\left[X_{(\eta+2):(\eta+2)}-X_{(\eta+1):(\eta+2)}\right]
\end{aligned}
$$




$$
\begin{aligned}
& \lambda_{3}^{\eta}=\frac{1}{3} E\left[X_{(\eta+3):(\eta+3)}-2 X_{(\eta+2):(\eta+3)}+X_{(\eta+1):(\eta+3)}\right] \\
& \lambda_{4}^{\eta}=\frac{1}{4} E\left[X_{(\eta+4):(\eta+4)}-3 X_{(\eta+3):(\eta+4)}+3 X_{(\eta+2):(\eta+4)}-X_{(\eta+1):(\eta+4)}\right]
\end{aligned}
$$

where $E\left[X_{(j):(r)}\right]$ is the expectation of the $j$-th order statistic of a sample of size $r$ drawn from a probability distribution $F(x)=\mathrm{P}(X \leq x)$, given by (Hosking 1990)

$$
E\left[X_{(j):(r)}\right]=\frac{r !}{(j-1) !(r-j) !} \int_{0}^{1} x(F) F^{j-1}(1-F)^{r-j} \mathrm{~d} F
$$

For the zero level, i.e., $\eta=0$, the LH-moments are identical to the L-moments defined in Hosking (1990). For $\eta=1,2, \ldots$, the LH-moments are respectively called L1-moments, L2-moments, and so on. As the level $\eta$ increases, the LH-moments increasingly reflect the characteristics of the upper part of the probability distributions and the large events in the sample data.

Analogous to the traditional moments and L-moments, the first four LH-moments, i.e., $\lambda_{1}^{\eta}, \lambda_{2}^{\eta}$, $\lambda_{3}^{\eta}$, and $\lambda_{4}^{\eta}$ represent population measures of location, scale, skewness, and kurtosis, respectively. Specifically, $\lambda_{1}^{\eta}$ denotes the mean, and $\lambda_{2}^{\eta}, \lambda_{3}^{\eta}$, and $\lambda_{4}^{\eta}$ respectively characterize the spreadness, asymmetry, and peakedness of the upper part of the probability distribution. The normalized LH-moments ratios, named LH-coefficient of variation (LH-CV), LH-skewness, and LH-kurtosis, are respectively defined as (Murshed et al. 2014)

LH-CV: $\quad \tau_{2}^{\eta}=\frac{\lambda_{2}^{\eta}}{\lambda_{1}^{\eta}}$

LH-skewness: $\tau_{3}^{\eta}=\frac{\lambda_{3}^{\eta}}{\lambda_{2}^{\eta}}$

LH-kurtosis: $\tau_{4}^{\eta}=\frac{\lambda_{4}^{\eta}}{\lambda_{2}^{\eta}}$

The LH-moments can be estimated theoretically by using the normalized probability weighted moments (PWM) (Hosking et al. 1985), defined as

$$
B_{r}=\frac{\int_{0}^{1} x(F) F^{r} \mathrm{~d} F}{\int_{0}^{1} F^{r} \mathrm{~d} F}=(r+1) \int_{0}^{1} x(F) F^{r} \mathrm{~d} F=(r+1) \beta_{r}
$$

where $\beta_{r}$ is the standard PWM (Greenwood et al. 1979). Substituting Eqs. (5) and (12) into Eqs. (1)-(4) yields the expressions of the theoretical relationships between the first four LH-moments and the 
normalized PWMs, given by

$\lambda_{1}^{\eta}=B_{\eta}$

$\lambda_{2}^{\eta}=\frac{1}{2 !}(\eta+2)\left[B_{\eta+1}-B_{\eta}\right]$

$\lambda_{3}^{\eta}=\frac{1}{3 !}(\eta+3)\left[(\eta+4) B_{\eta+2}-2(\eta+3) B_{\eta+1}+(\eta+2) B_{\eta}\right]$

$\lambda_{4}^{\eta}=\frac{1}{4 !}(\eta+4)\left[(\eta+6)(\eta+5) B_{\eta+3}-3(\eta+5)(\eta+4) B_{\eta+2}+3(\eta+4)(\eta+3) B_{\eta+1}-(\eta+3)(\eta+2) B_{\eta}\right]$

Given a cumulative probability distribution $F(x)$ such as the Weibull distribution, a quantile function $x(F)$ is available, and the normalized PWMs at different orders can be calculated by using Eq. (9). Subsequently, the first four LH-moments can be estimated using Eqs. (10)-(13).

In addition to the theoretical LH-moments estimated by using the normalized PWM, the sample LH-moments can be computed from the sample order statistics directly without the need to know the probability distribution. Wang [1997] developed the following equations as direct unbiased estimators of the first four sample LH-moments at the $\eta$-th level:

$$
\begin{aligned}
& \hat{\lambda}_{1}^{\eta}=\frac{1}{C_{N}^{\eta+1}} \sum_{i=1}^{N} C_{i-1}^{\eta} x_{(i)} \\
& \hat{\lambda}_{2}^{\eta}=\frac{1}{2 C_{N}^{\eta+2}} \sum_{i=1}^{N}\left(C_{i-1}^{\eta+1}-C_{i-1}^{\eta} C_{N-i}^{1}\right) x_{(i)} \\
& \hat{\lambda}_{3}^{\eta}=\frac{1}{3 C_{N}^{\eta+3}} \sum_{i=1}^{N}\left(C_{i-1}^{\eta+2}-2 C_{i-1}^{\eta+1} C_{N-i}^{1}+C_{i-1}^{\eta} C_{N-i}^{2}\right) x_{(i)} \\
& \hat{\lambda}_{4}^{\eta}=\frac{1}{4 C_{N}^{\eta+4}} \sum_{i=1}^{N}\left(C_{i-1}^{\eta+3}-3 C_{i-1}^{\eta+2} C_{N-i}^{1}+3 C_{i-1}^{\eta+1} C_{N-i}^{2}-C_{i-1}^{\eta} C_{N-i}^{3}\right) x_{(i)}
\end{aligned}
$$

where $x_{(i)}, i=1,2, \cdots, N$ are sample values ranked in ascending order and $N$ represents the sample size. The binomial coefficient is written as

$C_{m}^{j}=\left(\begin{array}{c}m \\ j\end{array}\right)=\frac{m !}{j !(m-j) !}$

denoting the number of combinations of any $j$ items from $m$ items. When $j>m$, the value is equal to zero. 


\section{Weibull Distribution and its LH-moments}

The probability density function (PDF) $f(x)$ of the complete three-parameter Weibull distribution (Izadparast and Niedzwecki 2010) is given by

$$
f(x)=\frac{\delta}{\kappa}\left(\frac{x-\xi}{\kappa}\right)^{\delta-1} \exp \left(-\left(\frac{x-\xi}{\kappa}\right)^{\delta}\right)
$$

where $x \geq \xi, \kappa>0, \delta>0$, and $\xi, \kappa$, and $\delta$ are the location, scale, and shape parameters, respectively. The cumulative distribution function (CDF) $F(x)$ and quantile function $x(F)$ (Izadparast and Niedzwecki 2010) are written as

$$
\begin{aligned}
& F(x)=1-\exp \left(-\left(\frac{x-\xi}{\kappa}\right)^{\delta}\right) \\
& x(F)=\xi+\kappa[-\ln (1-F)]^{1 / \delta}
\end{aligned}
$$

In Eqs. (19)-(21), let the location parameter be $\xi=0$ or consider $y=x-\xi$ when $\xi$ is a known value; the three-parameter Weibull distribution then reduces to the two-parameter Weibull distribution.

The parameters of the Weibull distributions can be estimated by using different methods. In the present study, the LH-moments method is proposed for parameter estimation as a generalization of the L-moments method. Specifying the quantile function of the three-parameter Weibull distribution in the normalized PWM, that is, substituting Eq.(21) into Eq.(9), yields

$$
\begin{aligned}
B_{r} & =\xi+(r+1) \kappa \int_{0}^{1}[-\ln (1-F)]^{1 / \delta} F^{r} \mathrm{~d} F \\
& =\xi+(r+1) \kappa \int_{0}^{\infty} z^{1 / \delta}[1-\exp (-z)]^{r} \exp (-z) \mathrm{dz}
\end{aligned}
$$

By applying the binomial theorem, the expression of the normalized PWM can be derived as

$$
B_{r}=\xi+\kappa \Gamma(1+1 / \delta) \sum_{j=0}^{r}(-1)^{j} C_{r+1}^{j+1}(j+1)^{-1 / \delta}
$$

where $\Gamma()$ is the Gamma function.

Further substituting Eq.(22) into Eqs.(10)-(13) yields the first four theoretical LH-moments of the three-parameter Weibull distribution, given by

$$
\begin{aligned}
& \lambda_{1}^{\eta}=\xi+\kappa \Gamma(1+1 / \delta) \sum_{j=0}^{\eta}(-1)^{j}(j+1)^{-1 / \delta} C_{\eta+1}^{j+1} \\
& \lambda_{2}^{\eta}=\frac{1}{2 !}(\eta+2) \kappa \Gamma(1+1 / \delta) \sum_{j=0}^{\eta+1}(-1)^{j}(j+1)^{-1 / \delta} C_{\eta+1}^{j}
\end{aligned}
$$




$$
\begin{aligned}
& \lambda_{3}^{\eta}=\frac{1}{3 !}(\eta+3) \kappa \Gamma(1+1 / \delta) \sum_{j=0}^{\eta+2}(-1)^{j}(j+1)^{-1 / \delta} C_{\eta+2}^{j}(j+2) \\
& \lambda_{4}^{\eta}=\frac{1}{4 !}(\eta+4) \kappa \Gamma(1+1 / \delta) \sum_{j=0}^{\eta+3}(-1)^{j}(j+1)^{-1 / \delta} C_{\eta+3}^{j}(j+2)(j+3)
\end{aligned}
$$

These equations denote the relationships between the LH-moments and the three parameters of the Weibull distribution, and they can be used for parameter estimation. For $\eta=0$, the equations will take the special form by using the L-moments, as given by

$$
\begin{aligned}
& \lambda_{1}=\xi+\kappa \Gamma(1+1 / \delta) \\
& \lambda_{2}=\kappa \Gamma(1+1 / \delta)\left(1-2^{-1 / \delta}\right) \\
& \lambda_{3}=\kappa \Gamma(1+1 / \delta)\left(1-3 \times 2^{-1 / \delta}+2 \times 3^{-1 / \delta}\right) \\
& \lambda_{4}=\kappa \Gamma(1+1 / \delta)\left(1-6 \times 2^{-1 / \delta}+10 \times 3^{-1 / \delta}-5 \times 4^{-1 / \delta}\right)
\end{aligned}
$$

\section{Parameter Estimation using LH-moments}

Given an ordered sample, the $\eta$-th level of the $r$-th order sample LH-moments, denoted by $\hat{\lambda}_{r}^{\eta}$, can be estimated directly by using Eqs.(14)-(17). Subsequently, by equating the theoretical LH-moments $\lambda_{r}^{\eta}$ in Eqs. (23)-(26) to the corresponding sample LH-moments $\hat{\lambda}_{r}^{\eta}$, the unknown parameters of the Weibull distribution can then be calculated.

First, the equation for the LH-skewness can be derived from Eqs. (15), (16), (24), and (25) and be written as

$$
\hat{\tau}_{3}^{\eta}=\frac{\hat{\lambda}_{3}^{\eta}}{\hat{\lambda}_{2}^{\eta}}=\tau_{3}^{\eta}=\frac{(\eta+3) \sum_{j=0}^{\eta+2}(-1)^{j}(j+1)^{-1 / \delta} C_{\eta+2}^{j}(j+2)}{3(\eta+2) \sum_{j=0}^{\eta+1}(-1)^{j}(j+1)^{-1 / \delta} C_{\eta+1}^{j}}
$$

Eq. (31) represents the relationship between the shape parameter $\delta$ and the LH-skewness $\tau_{3}^{\eta}$. By specifying $\delta=1$ in Eq. (31), a uniform value of $\tau_{3}^{\eta}$ can be obtained as

$\tau_{3}^{\eta}=1 / 3 \quad$ when $\delta=1$ 
This reveals that the LH-skewness is constant at all levels when $\delta=1$.

Eq. (31) can thus be used to estimate the shape parameter $\delta$ but not to calculate it directly. An iterative procedure needs to be performed to obtain an approximate solution. The required tolerance for convergence needs to be specified, such as $10^{-4}$ in the present study, for minimizing the following discrepancy between the calculated and sample LH-skewness:

$f(\delta)=\left|\tau_{3}^{\eta}-\hat{\tau}_{3}^{\eta}\right|$

Upon subsequently applying the approximate shape parameter $\delta$, the scale parameter can be calculated by equating the $2^{\text {nd }} \mathrm{LH}-$ moments between Eqs. (15) and (24)

$$
\kappa=\frac{2 \hat{\lambda}_{2}^{\eta}}{(\eta+2) \Gamma(1+1 / \delta) \sum_{j=0}^{\eta+1}(-1)^{j}(j+1)^{-1 / \delta} C_{\eta+1}^{j}}
$$

Finally, the location parameter can be calculated by the following equation derived from Eqs. (14) and (23):

$\xi=\hat{\lambda}_{1}^{\eta}-\kappa \Gamma(1+1 / \delta) \sum_{j=0}^{\eta}(-1)^{j}(j+1)^{-1 / \delta} C_{\eta+1}^{j+1}$

For the two-parameter Weibull distribution, the location parameter is set as $\xi=0$, and the unknown shape and scale parameters can be estimated by using Eqs. (31) and (33) similarly. Specifically, for $\eta=$ 0 , the equations for parameter estimation using L-moments are then derived.

\section{Evaluation of Goodness-of-fit}

The LH-kurtosis is not employed to estimate the three parameters of the Weibull distribution directly, and it can be used to assess the accuracy of the parameter estimation. The relative discrepancy between the theoretical LH-kurtosis using the estimated parameters and the sample LH-kurtosis using the sample data is written as

$\mathrm{ERR}_{\mathrm{kur}}=\frac{\left|\tau_{4}^{\eta}-\hat{\tau}_{4}^{\eta}\right|}{\hat{\tau}_{4}^{\eta}} \times 100 \%$

where $\tau_{4}^{\eta}=\lambda_{4}^{\eta} / \lambda_{2}^{\eta}$ is the theoretical LH-kurtosis and $\hat{\tau}_{4}^{\eta}=\hat{\lambda}_{4}^{\eta} / \hat{\lambda}_{2}^{\eta}$ is the sample LH-kurtosis. The relative discrepancy is normally expressed as a percentage value. 
In addition, the root-mean-square error (RMSE) of the estimated quantiles is used to quantify the tolerance between the measured data and the model estimates by the Weibull distribution. Thus, for a random process

$$
\mathrm{RMSE}=\sqrt{\frac{1}{N} \sum_{i=1}^{N}\left[x\left(F_{i}\right)_{\mathrm{data}}-x\left(F_{i}\right)_{\text {estimated }}\right]^{2}}
$$

where $N$ is the size of the sample, and $x\left(F_{i}\right)$ is the quantile function with a cumulative probability of

$$
F_{i}=n_{i} /(N+1)
$$

where $n_{i}$ is the number of sample values, which is no more than $x_{(i)}$.

As mentioned earlier, the large extreme values with small probabilities of exceedance are usually of the greatest concern in practice. Therefore, rather than the overall tolerance using all quantiles, the tail RMSE is evaluated similarly by considering only the large sample values with probability of exceedance $\left(1-F_{i}\right) \leq 0.1$. In the application to random waves and wave run-ups, the RMSE values are usually normalized by using the significant wave height $H_{s}$, allowing the error estimate to be interpreted as a percentage of error relative to the specified wave height.

\section{Experiment on Wave Run-ups of a Deepwater Semi-submersible Platform}

\section{Description of Experiment}

An experimental study on the wave run-ups of a deepwater semi-submersible platform was conducted in the Deepwater Offshore Basin of Shanghai Jiao Tong University in China. The basin has length, width, and maximum water depth of 50, 40, and $10 \mathrm{~m}$, respectively. A large-area movable floor allows the flexible modeling of water depth from 0 to $10 \mathrm{~m}$ as required. A secondary movable floor in the deep pit with 5-m diameter further allows the modeling of water depth within 10 and $40 \mathrm{~m}$. Various ocean environments can be modeled, including collinear and non-collinear waves, currents and winds simulated by using two multiflap wave generators, a deepwater global current generation system, and an axial wind fans matrix.

The semi-submersible model consists of four rectangular columns with sloping tops, two pontoons, and two rectangular bracings interconnecting the pontoons. The survival condition is considered in the 
present study of wave run-ups. The operational condition is not included here since more severe wave run-ups under survival conditions must be considered for the air-gap issue. Table 1 shows the main particulars of the semi-submersible platform under survival condition with a linear scale ratio of 1:60 between the prototype and the model. Fig.1 shows the model in the basin.

The design water depth of the semi-submersible platform is $1500 \mathrm{~m}$. The spread mooring system of the semi-submersible platform consists of 16 chain-wire-chain mooring lines that are grouped into 4 bundles. Each group has 4 mooring lines, and the interval angle is $4^{\circ}$. In terms of the scale ratio of 1:60 and the maximum water depth of the wave basin, a truncated mooring system was designed and modeled according to the truncated water depth of $600 \mathrm{~m}$. The principal properties of the semi-submersible model and truncated mooring system, such as the locations of Centre of Gravity (COG), radii of gyration, natural periods, and horizontal restoring curves, were validated via comparison with the target values prior to the wave tests.

Corresponding to the survival condition, typical 100-y return environments were applied, consisting of a random wave with a significant wave height of $H_{s}=15.0 \mathrm{~m}$ and peak wave period of $T_{p}=15.1 \mathrm{~s}$, as well as collinear steady wind with mean wind speed of $V_{w}=49.5 \mathrm{~m} / \mathrm{s}$ and uniform current with mean surface current speed of $V_{c}=1.95 \mathrm{~m} / \mathrm{s}$. The typical JONSWAP wave spectrum is applied to simulate the random waves, with a peakedness factor of $\gamma=2.4$. The time duration of each irregular wave realization is $\sim 23.2 \mathrm{~min}$ in the model scale, corresponding to $3 \mathrm{~h}$ in the full scale.

\section{Experimental Data}

In the experiment, the data sampling frequency was $25 \mathrm{~Hz}$ and the data measurements included the six degrees-of-freedom (DOF) motions, top tensions of all mooring lines, translational accelerations, and relative wave motions. For the probability analysis of air gap response, the present paper focuses on the relative wave motions which are measured using a number of resistance-type wave probes. Fig.2 shows a plan view of the tested semi-submersible platform model and wave probe locations. Three wave headings were considered, including head, quartering, and beam seas. Wave probe 1 was installed at the mid of two forward columns in head and quartering seas and mid of two lateral columns in beam seas to measure the upstream wave run-ups. Wave probes 2 and 3 were installed in the vicinity of the fore column and wave probes 4 and 5 were surrounding the aft column. Wave probe 6 was installed at 
the center of the moon pool and the platform. Wave probes 1,2,3, and 6 are out of deck area and distorted waves can probably run up to above the deck, resulting in larger measurements than the still water air gap. Nevertheless, wave probes 4 and 5 are located within the deck area and thus the measurements will be theoretically upper limited to zero air gap value by the deck bottom. Before placing the model in the basin, the specified irregular wave was calibrated along with the current such that the resulting spectrum closely matches the corresponding target, as shown in Fig. 3. The good agreement indicates that high-quality wave generation has been achieved. The time series of the calibrated wave is further used for statistical analysis as the incident wave.

\section{Probability Analysis Results and Discussion}

\section{Basic Statistics}

Before conducting the probability analysis of wave run-ups, the measured time series of the relative wave motions was analyzed, some of the basic statistics of which are summarized in Table 2, along with that of the incident wave for comparison. Here, $\sigma$ is the standard deviation; $T_{z}$, is the mean zero-up-crossing period; $N_{c}$, is the number of wave cycles representing the sample size for the probability analysis; $r_{3}$, is the coefficient of skewness. As a measure of the asymmetry of the probability distribution, the skewness is close to zero for a Gaussian distribution and an increase in the skewness indicates that there is more nonlinearity. Upon comparing the basic statistics for wave run-ups to those of the incident waves, the effects of the incident wave passing over the semi-submersible platform can be observed. The skewness values at locations WP4 and WP5 are significantly larger, indicating that the wave run-ups surrounding the aft columns are strongly nonlinear owing to wave-hull interactions. At the other upstream locations, including WP1 and WP3 at head seas, WP1-WP3 at quartering seas, and WP1 and WP2 at beam seas, the skewness values increase clearly and the wave run-ups exhibit more non-linearity. Furthermore, these nonlinear wave run-ups may result in wave breaking phenomenon, i.e. foaming on the crests, as observed in the vicinity of the semi-submersible during the experiment. In addition, the increased wave cycles along with the shortening of the mean zero-up-crossing periods also indicate the increased non-linearity of the wave run-ups owing to wave-hull interactions. The variation of the standard deviation depends on the 
location and heading. At upstream locations close to the columns, including WP2 and WP5 at head seas, WP2-WP5 at quartering seas, and WP3 and WP4 at beam seas, the standard deviations increase evidently, indicating enhanced wave run-ups due to the columns. At other locations, the standard deviations are smaller owing to the compliant motions of the semi-submersible platform. Moreover, wave breaking and loss of energy may also result in the reduction in the standard deviations of wave run-ups.

The vessel vertical motions of the compliant platform may significantly influence the wave run-ups (Izadparast and Niedzwecki 2010). Table 3 lists standard deviations of heave, roll, and pitch at the COG and vessel vertical motions at six wave probes. In comparison with the measurements of wave run-ups listed in Table 2, it can be seen that the magnitude of vessel vertical motions is considerable and tends to be an important factor affecting relative wave elevations.

\section{Goodness-of-fit Evaluation}

From basic statistical analysis of the measured time series, a set of crests of wave run-ups are obtained and ranked in ascending order for further probability analysis using the LHME. However, one of the difficulties of using the LHME in practical applications is the selection of $\eta$. In general, one needs to estimate the model parameters for a range of $\eta$ values and then select the optimum $\eta$ value through goodness-of-fit tests of probability distributions (Wang 1998). To evaluate the dependence of the goodness-of-fit on the level of LH-moments, $\eta$ values ranging from 0 to 30 with an interval of 1 are adopted to perform the analysis. The level cannot be set too large, because the binominal coefficient represented by Eq. (18) grows exponentially with it and may result in calculation instabilities at too high levels.

As one representation of the goodness-of-fit to the sample data, the relative error between the estimated and the sample LH-kurtosis is calculated using Eq. (35). As other representation, the tail RMSE between the estimated quantiles and the sample data is calculated using Eq. (36) and normalized by $H_{s}$. Fig. 4 shows the results for six wave run-ups and three test cases.

The variation with the $\eta$ level of LH-moments suggests that the relative error of LH-kurtosis and the relative tail RMSE have different trends. The tail RMSE decreases monotonically with an increase in the level, whereas the error of the LH-kurtosis fluctuates and a minimum value usually exists. 
Compared with the results estimated using L-moments, i.e., LH-moments at $\eta=0$, the minimum errors at higher levels of LH-moments tend to be smaller, leading to better estimation of the probability distributions. Furthermore, at the appropriate level corresponding to the minimum error of LH-kurtosis, both the error of LH-kurtosis and the tail RMSE are evidently small, as shown in Table 4. This indicates that the Weibull distribution model estimated using high-level LH-moments can well represent the probability distributions of extreme wave run-ups rather than that using L-moments. Moreover, an appropriate level can be determined for the probability analysis based on the minimum relative error of the LH-kurtosis, resulting in low tail RMSE as well. Therefore, goodness-of-fit tests on the basis of comparison of sample LH-kurtosis estimates and theoretical LH-kurtosis values of the fitted distributions using LH-moments are recommended as a procedure for selecting an optimum $\eta$, similar to the approximate goodness-of-fit tests for GEV distributions (Wang 1998). The estimates of Weibull parameters for the selected $\eta$ value and $\eta=0$ are listed in Table 5 .

\section{Wave Run-up Distributions using LH-moments}

Using the estimated parameters of the Weibull distributions, the exceedance probability function $1-F(x)$ at different levels of LH-moments can be obtained and compared to the measured data calculated by applying Eq. (37) to the sample data directly. Among the total levels from 0 to 30, four typical levels are selected to represent the comparisons with the measured data, one of which is the appropriate level corresponding to the minimum error of LH-kurtosis listed in Table 4. In addition, the level $\eta=0$ denotes the L-moments used for the estimation. As an example, Fig.5 shows the results of the wave run-ups at the center location WP6.

Along with the increasing level, the estimated probability distributions clearly tend to increasingly match the upper part of the measured data with small probabilities of exceedance, whereas they deviate gradually from the lower part of the data with large probabilities of exceedance. In particular, at an appropriate level with minimum error of LH-kurtosis, the estimated probability distribution agrees well with the measured data at the upper part of large quantiles, but it shows poor agreement with the lower part of small quantiles. In contrast, the estimated distributions based on L-moments, i.e., $\eta=0$, are noticeably different from the measured data of large wave run-ups, which are typically of more interest. This indicates that the estimation using LH-moments at an appropriate level can well represent the 
probability distribution of large wave run-ups.

Further for evaluating the variability of the predictions from different methods, three realizations of incident waves with different seeds were measured in the wave basin and the sample data of wave crest heights were used for model estimates. Fig. 6 shows the comparison between the measured data and the exceedance probability distributions estimated by using L-moments and LH-moments at the appropriate level. It is observed that the extreme samples and the model estimates from three realizations show slight variability and similar prediction using both methods. The difference of the upper part of probability distributions between using LH-moments and L-moments is less clear than those of wave run-ups.

In terms of the probability analysis based on the Weibull distribution model for all wave run-ups at different locations and wave headings, those estimated using LH-moments at the appropriate levels are derived and compared with the measured data, as shown in Fig. 7. Note that only the upper part of large wave run-ups is of interest, and the locations are shown in Fig. 2, where WP1 is between upstream columns, WP2 and WP3 are close to the fore column, WP4 and WP5 are close to the aft column, and WP6 is located at the platform center.

Fig. 7 clearly shows the dependence of wave run-up distributions on the location and wave heading. The measured data of WP1 and WP6 is smaller than the still water air gap of $18 \mathrm{~m}$, and the estimated distribution shows good agreement with it. For WP2-WP5, which surround the columns, the extreme data measured is larger than that for the still-water air gap and, in this case, shows poor agreement with the estimated distribution. For extreme wave run-ups at WP4 and WP5, the water surface reaches the underside of the lower deck and wave impact occurs. Wave run-up is blocked and distorted, resulting in different probability characteristics and further leading to the evident discrepancy between the Weibull distribution and the measured data.

The wave heading is found significantly influencing the wave run-ups, especially around the columns. In head seas, the wave run-ups at WP2 and WP5 are clearly higher than the others, indicating that the fore and aft columns reflect the incoming waves and the water surface climbs up along the front column side significantly. In addition, both wave-induced heave and pitch motions result in considerable vessel vertical motions at the columns and thus also play an important role in forming the wave run-ups. With regard to the wave run-ups at WP5 surrounding the aft column, the extreme measured data is lower than the estimated distributions owing to the blockage of the underside of the 
deck. In fact, it is shown that the wave run-up at WP5 is more serious than that at WP2 owing to the wave interactions between the aft and the fore columns. The wave run-ups at WP3 and WP4 along the lateral column side are relatively lower because the diffracted waves can easily pass away from the lateral column sides.

Beam seas are similar to head seas in that the wave run-ups at WP3 and WP4 in front of the columns are clearly higher than the others owing to wave reflection and running up effects by the columns. Effect of wave-induced heave and roll motions is also considerable. At the lateral column sides, the wave run-up at WP2 is evidently smaller than that at WP5. The wave run-up at WP1 is slightly higher than that in head and quartering seas owing to the greater influence of the submerged pontoon than that of the submerged bracing.

In quartering seas, the wave run-ups at WP2 and WP3 surrounding the fore column are higher than the others, and their probability distributions are almost consistent. In comparison, the wave run-ups at WP4 and WP5 surrounding the aft column are evidently smaller, indicating the slighter influence of wave interactions between columns, because the distorted waves between lateral columns can be transmitted downstream more easily. However, the wave run-up at WP6 is clearly higher than that in head seas and beam seas owing to the wave interaction between two diagonal columns along the incident wave direction. Of course, it is significantly lower compared to the incident wave in a manner similar to the basic statistics, indicating the positive influence of the compliant motions of the semi-submersible platform.

The wave run-ups in front of the columns along the wave incoming direction, especially the aft columns, show significantly larger probability distributions than those at other locations. This clearly demonstrates that the surrounding columns, especially the aft columns, are more likely to suffer from negative air gap and wave impact incidents than the other platform areas.

\section{Air Gap and Probability of Exceedance}

From the viewpoint of design, the exceedance probability of the wave run-up above the specified still-water air gap is a safety index for the evaluation of the air gap issue. Based on the Weibull distributions of the wave run-ups, the exceedance probabilities corresponding to the still-water air gap are further estimated for different locations, and the results are presented in Fig. 8. The result of WP6 at 
the platform center in head seas is not shown in the figure because the probability is too small $\left(\sim 10^{-10}\right)$.

The quantitative probabilities clearly show a similar trend as the previous analysis of probability distributions. The locations WP5 and WP2 in head seas; WP4 and WP3 in beam seas; WP2, WP3, and WP5 in quartering seas have higher probabilities of suffering from negative air gap issues. All these locations are in front of the columns along the wave incoming direction.

On the other hand, the maximum wave run-ups with a particular probability of exceedance, such as that in a 3-h duration, are helpful for designers to determine the still-water air gap and assess the possible wave impact of the platform structure. In terms of the zero-up-crossing period, the total wave cycles in $3 \mathrm{~h}$ can be counted, and then, the probability of exceedance corresponding to the maximum value can be calculated by Eq. (37) by setting $n_{i}=1$. Subsequently, the maximum quantile with the calculated probability of exceedance, commonly called the 3-h estimated extreme, can be derived by using the Weibull distribution model estimated by the LH-moments at the appropriate level.

Fig. 9 shows the results of the 3-h estimated extremes using LH-moments at the appropriate level. Compared with the still-water air gap, the 3-h estimated extremes are significantly larger at WP5 and WP2 in head seas; WP4, WP3, and WP5 in beam seas; and WP2, WP3, and WP5 in quartering seas. In particular, in quartering and beam seas, the largest increments are more than $50 \%$, leading to quite serious wave impact on the deck. It is difficult to increase the still-water air gap to a great extent in the design, and thus, it is necessary to reliably assess the wave impact to determine the deck structure strength. The extremes quantitatively estimated through probability analysis tend to be the proper reference to assess the probable wave impact loads.

\section{Conclusions}

The three-parameter Weibull distribution is applied to probability analyses of the wave run-ups of a deepwater semi-submersible platform under survival conditions by using the LH-moments method for parameter estimation. Explicit relationships between the first three LH-moments at arbitrary levels and the parameters of the Weibull distribution are established analytically. The accuracy of LH-kurtosis estimation is proposed to determine the appropriate level for probability analysis.

The probability distributions based on parameter estimation using high-level LH-moments can well represent the large wave run-ups compared to that using L-moments. The LH-kurtosis estimation has a 
minimum relative error, and the corresponding tail RMSE is low. The probability distribution depends on the location and heading, especially around the columns, where the wave run-ups are significantly higher than others. In front of the columns along the wave incoming direction, especially the aft column, the wave run-ups show the highest probability distributions. This means that the surrounding columns, especially the aft columns, are more likely to suffer from negative air gap issues and wave impact incidents than the other platform areas. At the platform center, the wave run-up is significantly lower than the incident wave owing to the positive influence of the compliant motions. The air gap estimates are more serious in quartering and beam seas than in head seas.

For extreme wave run-ups beyond the still-water air gap, however, less agreement is observed between the estimated probability distribution and the measured data because the water surface reaches the underside of the deck, resulting in distorted waves with different characteristics. Both measurement methods and probability analyses warrant further research.

\section{Acknowledgments}

The authors would like to acknowledge the support of the National Natural Science Foundation of China (Grant Nos. 51279104 and 51239007) and the project of the CNOOC-SJTU Deepwater Engineering Research Center. The authors are grateful to the anonymous reviewers whose valuable comments and suggestions improved the manuscript.

\section{References}

Al-Humoud, J., Tayfun, M. A., and Askar, H. (2002). "Distribution of nonlinear wave crests." Ocean engineering, 29(15), 1929-1943.

Bobée, B., and Rasmussen, P. F. (1995). "Recent advances in flood frequency analysis." Reviews of Geophysics, 33(S2), 1111-1116.

Forristall, G. Z. (2007). "Wave crest heights and deck damage in hurricanes Ivan, Katrina, and Rita." Proc., 22nd Offshore Technology Conference, ASME, Houston, USA, OTC 18620.

Franco, C., and Franco, L. (1999). "Overtopping formulas for caisson breakwaters with nonbreaking 3D waves." Journal of Waterway, Port, Coastal, and Ocean Engineering, 125(2), 98-108. 
Greenwood, J. A., Landwehr, J. M., Matalas, N. C., and Wallis, J. R. (1979). "Probability weighted moments: Definition and relation to parameters of distribution expressible in inverse form". Water resources research, 15(5), 1049-1054.

Hewa, G. A., Wang, Q. J., McMahon, T. A., Nathan, R. J., and Peel, M. C. (2007). "Generalized extreme value distribution fitted by LH moments for low - flow frequency analysis." Water resources research, 43(6), W06301 1-10.

Hosking, J. R. M., Wallis, J. R., and Wood, E. F. (1985). "Estimation of the generalized extreme value distribution by the method of probability weighted moments." Technometrics, 27(3), 251-261.

Hosking, J. R. M. (1990). "L-moments: analysis and estimation of distributions using linear combinations of order statistics." Journal of the Royal Statistical Society. Series B (Methodological), 105-124.

Indrebo, A. K., and Niedzwecki, J. M. (2004). "Wave runup on cylinders subject to deep water random waves." Proc., 23rd International Conference on Offshore Mechanics and Arctic Engineering, ASME,Vancouver, Canada, 385-392.

Izadparast, A. H., and Niedzwecki, J. M. (2009). "Estimating wave crest distributions using the method of L-moments." Applied Ocean Research, 31(1), 37-43.

Izadparast, A. H., and Niedzwecki, J. M. (2010). "Probability distributions of wave run-up on a TLP model." Marine Structures, 23(2), 164-186.

Kleefsman, K. M. T., Fekken, G., Veldman, A. E. P., Iwanowski, B., and Buchner, B. (2005). "A volume-of-fluid based simulation method for wave impact problems." Journal of Computational Physics, 206(1), 363-393.

Kriebel, D. L., and Dawson, T. H. (1991). "Nonlinear effects on wave groups in random seas." Journal of Offshore Mechanics and Arctic Engineering, 113(2), 142-147.

Martin, A. J., Easson, W. J., and Bruce, T. (2001). "Runup on columns in steep, deep water regular waves." Journal of waterway, port, coastal, and ocean engineering, 127(1), 26-32.

Mathiesen, M., Goda, Y., Hawkes, P. J., Mansard, E., Martín, M. J., Peltier, E., Thompson, E. F., and Van Vledder, G. (1994). "Recommended practice for extreme wave analysis." Journal of hydraulic Research, 32(6), 803-814.

Matsumoto, F. T., Watai, R. A., Simos, A. N., and Ferreira, M. D. A. S. (2013). "Wave Run-Up and Air Gap Prediction for a Large-Volume Semi-Submersible Platform." Journal of Offshore Mechanics 
and Arctic Engineering, 135(1), 011302.

Meshgi, A., and Khalili, D. (2009). "Comprehensive evaluation of regional flood frequency analysis by L-and LH-moments. II. Development of LH-moments parameters for the generalized Pareto and generalized logistic distributions." Stochastic Environmental Research and Risk Assessment, 23(1), $137-152$

Murshed, M. S., Am Seo, Y., and Park, J.-S. (2014). "LH-moment estimation of a four parameter kappa distribution with hydrologic applications." Stochastic Environmental Research and Risk Assessment, 28(2), 253-262.

Nagatsuka, H., Kamakura, T., and Balakrishnan, N. (2013). "A consistent method of estimation for the three-parameter Weibull distribution." Computational Statistics \& Data Analysis, 58, 210-226.

Niedzwecki, J. M., and Duggal, A. S. (1992). "Wave runup and forces on cylinders in regular and random waves." Journal of waterway, port, coastal, and ocean engineering, 118(6), 615-634.

Niedzwecki, J. M., Van de Lindt, J. W., Gage, J. H., and Teigen, P. S. (2000). "Design estimates of surface wave interaction with compliant deepwater platforms." Ocean engineering, 27(8), $867-888$.

Nielsen, F. G. (2003). "Comparative study on airgap under floating platforms and run-up along platform columns." Marine structures, 16(2), 97-134.

Priyanto, A., Maimun, A., Kader, A.S.A., Nasrudin, I., Ghani, M.P.A., Nur, I., and Jaswar, K. (2014). " Probability distribution of wave run up and dynamic response on a large volume semi-submersible." Ocean Engineering, 76, 52-64.

Shan, T.-B., Yang, J.-M., Li, X., and Xiao, L.-F. (2011). "Experimental investigation on wave run-up characteristics along columns and air gap response of semi-submersible platform." Journal of Hydrodynamics, Ser. B, 23(5), 625-636.

Simos, A. N., Fujarra, A. L. C., Sparano, J. o. V., Umeda, C. H., and Rossi, R. R. (2006). "Experimental evaluation of the dynamic air gap of a large-volume semi-submersible platform." Proc., 25th International Conference on Offshore Mechanics and Artic Engineering, ASME, Hamburg, Germany, 393-399.

Simos, A. N., Sparano, J. o. V., Aranha, J. A. P., and Matos, V. c. L. F. (2008). "2nd order hydrodynamic effects on resonant heave, pitch and roll motions of a large-volume semi-submersible platform." Proc., 27th International Conference on Offshore Mechanics and Artic Engineering, ASME, 
Estoril, Portugal, 229-237.

Stansberg, C. T., Baarholm, R., Kristiansen, T., Hansen, E. W. M., and Rortveit, G. (2005). "Extreme wave amplification and impact loads on offshore structures." Proc., 20th Offshore Technology Conference, ASME, Houston, USA, OTC17487.

Veldman, A. E. P., Luppes, R., Bunnik, T., Huijsmans, R. H. M., Duz, B., Iwanowski, B., Wemmenhove, R., Borsboom, M. J. A., Wellens, P. R., and van der Heiden, H. J. L. (2011). "Extreme wave impact on offshore platforms and coastal constructions." Proc., 30th International Conference on Ocean, Offshore and Arctic Engineering, ASME, Rotterdam, The Netherlands, 365-376.

Wang, Q. J. (1997). "LH moments for statistical analysis of extreme events." Water Resources Research, 33(12), 2841-2848.

Wang, Q. J. (1998). "Approximate goodness-of-fit tests of fitted generalized extreme value distributions using LH moments." Water Resources Research, 34(12), 3497-3502.

Winterstein, S. R., and Sweetman, B. (2001). "Air Gap Response of Floating Structures: Statistical Predictions Versus Observed Behavior." Journal of Offshore Mechanics and Arctic Engineering, 123(3), 118-123. 
Table 1.

Main particulars of semi-submersible platform under survival conditions

\begin{tabular}{lccc}
\hline \multicolumn{1}{c}{ Parameters } & Units & Prototype & Model \\
\hline Pontoon dimensions & $\mathrm{m}$ & $111 \times 20 \times 11$ & $1.85 \times 0.333 \times 0.183$ \\
Column dimensions & $\mathrm{m}$ & $22.5 \times 16 \times 40$ & $0.375 \times 0.267 \times 0.667$ \\
Draft & $\mathrm{m}$ & 33.0 & 0.55 \\
Still water air-gap & $\mathrm{m}$ & 18.0 & 0.3 \\
Displacement weight & $\mathrm{t}$ & 94920 & 0.4287 \\
Vertical center of gravity & $\mathrm{m}$ & 27.44 & 0.4573 \\
Roll radius of gyration & $\mathrm{m}$ & 37.75 & 0.6292 \\
Pitch radius of gyration & $\mathrm{m}$ & 40.72 & 0.6787 \\
Roll natural period & $\mathrm{s}$ & 42.3 & 5.46 \\
Pitch natural period & $\mathrm{s}$ & 35.3 & 4.56 \\
\hline
\end{tabular}




\section{Table 2.}

Basic statistics of wave run-ups and incident wave

\begin{tabular}{cccccccccccccc}
\hline Wave & \multicolumn{3}{c}{ Head seas } & \multicolumn{1}{c}{ Quartering seas } & \multicolumn{5}{c}{ Beam seas } \\
run-up & $\sigma(\mathrm{m})$ & $N_{c}$ & $T_{z}(\mathrm{~s})$ & $r_{3}$ & $\sigma(\mathrm{m})$ & $N_{c}$ & $T_{z}(\mathrm{~s})$ & $r_{3}$ & $\sigma(\mathrm{m})$ & $N_{c}$ & $T_{z}(\mathrm{~s})$ & $r_{3}$ \\
\hline WP1 & 3.47 & 1228 & 8.98 & 0.194 & 3.24 & 1224 & 8.98 & 0.263 & 3.57 & 1156 & 9.51 & 0.316 \\
WP2 & 3.88 & 1148 & 9.61 & 0.008 & 4.24 & 1071 & 10.26 & 0.241 & 3.24 & 1137 & 9.67 & 0.208 \\
WP3 & 2.85 & 1088 & 10.14 & 0.306 & 4.16 & 1105 & 9.95 & 0.244 & 4.41 & 1138 & 9.66 & 0.002 \\
WP4 & 3.09 & 1089 & 10.12 & 0.611 & 3.77 & 1106 & 9.94 & 0.609 & 4.45 & 1158 & 9.49 & 0.748 \\
WP5 & 4.38 & 1122 & 9.83 & 0.640 & 3.82 & 1089 & 10.09 & 0.793 & 3.20 & 1117 & 9.84 & 0.874 \\
WP6 & 3.05 & 1184 & 9.32 & 0.035 & 3.44 & 1143 & 9.61 & 0.369 & 3.09 & 1230 & 8.94 & -0.050 \\
Incident & 3.65 & 940 & 11.73 & 0.120 & 3.65 & 929 & 11.81 & 0.122 & 3.65 & 929 & 11.81 & 0.122 \\
\hline
\end{tabular}


Table 3.

Standard deviations of vessel vertical motions at COG and measurement locations of wave run-ups

\begin{tabular}{cccccccccc}
\hline Wave & Heave & Roll & Pitch & \multicolumn{5}{c}{ Vessel vertical motions (m) } \\
direction & $(\mathrm{m})$ & $(\mathrm{deg})$ & $(\mathrm{deg})$ & WP1 & WP2 & WP3 & WP4 & WP5 & WP6 \\
\hline Head & 1.44 & 0.70 & 1.41 & 1.82 & 1.86 & 1.73 & 1.80 & 1.60 & 1.44 \\
Quartering & 1.56 & 1.43 & 1.17 & 1.65 & 2.11 & 2.11 & 2.20 & 2.17 & 1.56 \\
Beam & 1.63 & 1.94 & 0.46 & 2.06 & 1.90 & 2.08 & 1.88 & 2.15 & 1.63 \\
\hline
\end{tabular}


Table 4.

Minimum errors of LH-kurtosis and corresponding levels as well as tail RMSEs

\begin{tabular}{cccccccccc}
\hline Wave & \multicolumn{3}{c}{ Head seas } & \multicolumn{3}{c}{ Quartering seas } \\
run-up & $\eta$ & Min ERRkur & Tail RMSE/Hs & $\eta$ & Min ERRkur & Tail RMSE/Hs & $\eta$ & Min ERRkur & Tail RMSE/Hs \\
\hline WP1 & 10 & 0.016 & 0.027 & 20 & 0.001 & 0.013 & 29 & 0.002 & 0.020 \\
WP2 & 30 & 0.046 & 0.027 & 20 & 0.002 & 0.052 & 9 & 0.002 & 0.011 \\
WP3 & 22 & 0.001 & 0.025 & 21 & 0.000 & 0.055 & 16 & 0.002 & 0.058 \\
WP4 & 4 & 0.004 & 0.009 & 24 & 0.002 & 0.012 & 6 & 0.010 & 0.064 \\
WP5 & 2 & 0.006 & 0.045 & 3 & 0.012 & 0.039 & 8 & 0.001 & 0.023 \\
WP6 & 26 & 0.001 & 0.010 & 17 & 0.001 & 0.019 & 23 & 0.001 & 0.013 \\
\hline
\end{tabular}


Table 5.

Estimates of Weibull parameters for $\eta=0$ and the selected $\eta$ value

\begin{tabular}{|c|c|c|c|c|c|c|c|c|c|c|c|c|}
\hline \multirow{2}{*}{$\begin{array}{l}\text { Wave } \\
\text { run-up }\end{array}$} & \multicolumn{4}{|c|}{ Head seas } & \multicolumn{4}{|c|}{ Quartering seas } & \multicolumn{4}{|c|}{ Beam seas } \\
\hline & $\eta$ & $\xi$ & $\kappa$ & $\delta$ & $\eta$ & $\xi$ & $\kappa$ & $\delta$ & $\eta$ & $\xi$ & $\kappa$ & $\delta$ \\
\hline \multirow[t]{2}{*}{ WP1 } & 0 & -0.882 & 5.601 & 1.970 & 0 & -0.998 & 5.400 & 1.946 & 0 & -0.796 & 5.670 & 1.648 \\
\hline & 10 & 2.360 & 2.341 & 1.069 & 20 & 3.957 & 0.891 & 0.702 & 29 & 4.237 & 1.518 & 0.833 \\
\hline \multirow[t]{2}{*}{ WP2 } & 0 & -0.536 & 5.553 & 1.793 & 0 & -0.372 & 6.010 & 1.707 & 0 & -0.404 & 4.845 & 1.668 \\
\hline & 30 & 5.396 & 0.665 & 0.611 & 20 & 5.832 & 0.550 & 0.514 & 9 & 0.777 & 3.664 & 1.368 \\
\hline \multirow[t]{2}{*}{ WP3 } & 0 & -0.280 & 4.193 & 1.700 & 0 & -0.184 & 5.489 & 1.589 & 0 & -0.086 & 5.683 & 1.591 \\
\hline & 22 & 3.551 & 0.742 & 0.654 & 21 & 5.219 & 0.691 & 0.548 & 16 & 4.633 & 1.187 & 0.651 \\
\hline \multirow[t]{2}{*}{ WP4 } & 0 & -0.616 & 5.663 & 1.828 & 0 & -0.403 & 6.096 & 1.600 & 0 & -0.127 & 6.010 & 1.387 \\
\hline & 4 & -0.351 & 5.388 & 1.757 & 24 & -2.099 & 7.824 & 1.937 & 6 & 2.244 & 3.568 & 0.984 \\
\hline \multirow[t]{2}{*}{ WP5 } & 0 & -0.296 & 6.328 & 1.467 & 0 & -0.150 & 5.851 & 1.425 & 0 & -0.339 & 5.256 & 1.439 \\
\hline & 2 & 0.099 & 5.889 & 1.384 & 3 & 0.502 & 5.123 & 1.284 & 8 & 1.595 & 3.281 & 1.049 \\
\hline \multirow[t]{2}{*}{ WP6 } & 0 & -0.591 & 4.787 & 1.966 & 0 & -0.648 & 5.449 & 1.813 & 0 & -0.282 & 4.290 & 1.738 \\
\hline & 26 & -42.99 & 46.30 & 11.29 & 17 & 3.403 & 1.862 & 0.973 & 23 & 1.685 & 2.327 & 1.127 \\
\hline
\end{tabular}




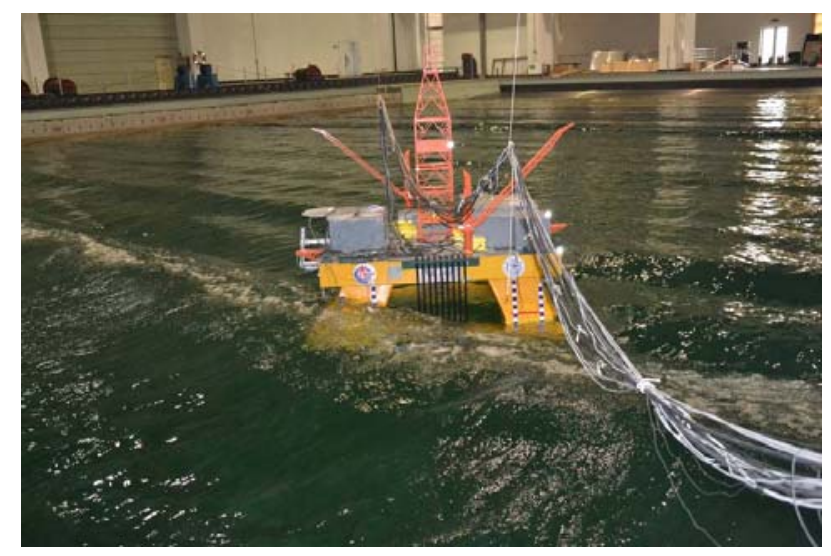

Fig. 1. Semi-submersible platform model in basin 


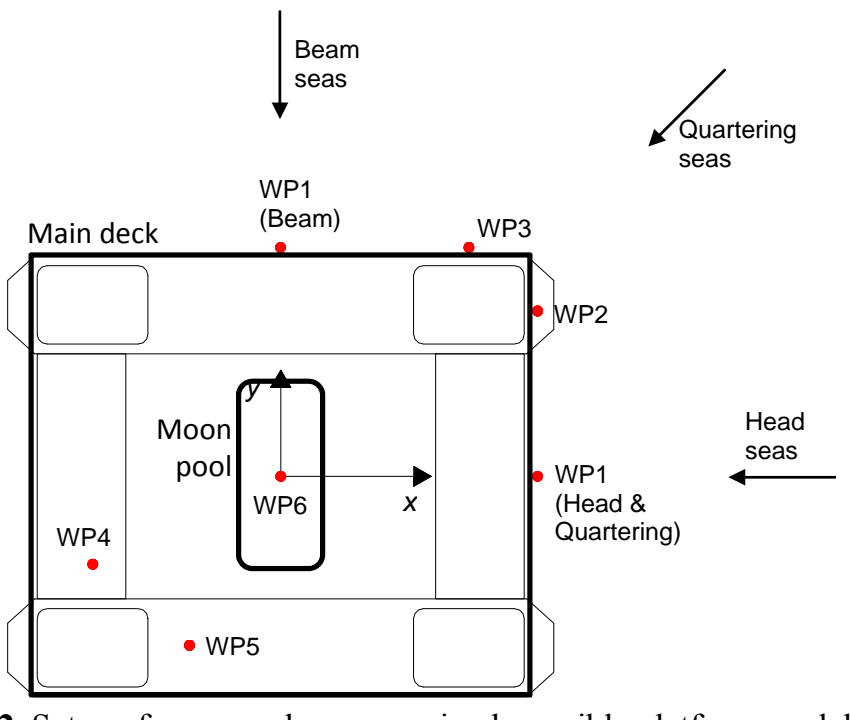

Fig. 2. Setup of wave probes on semi-submersible platform model 


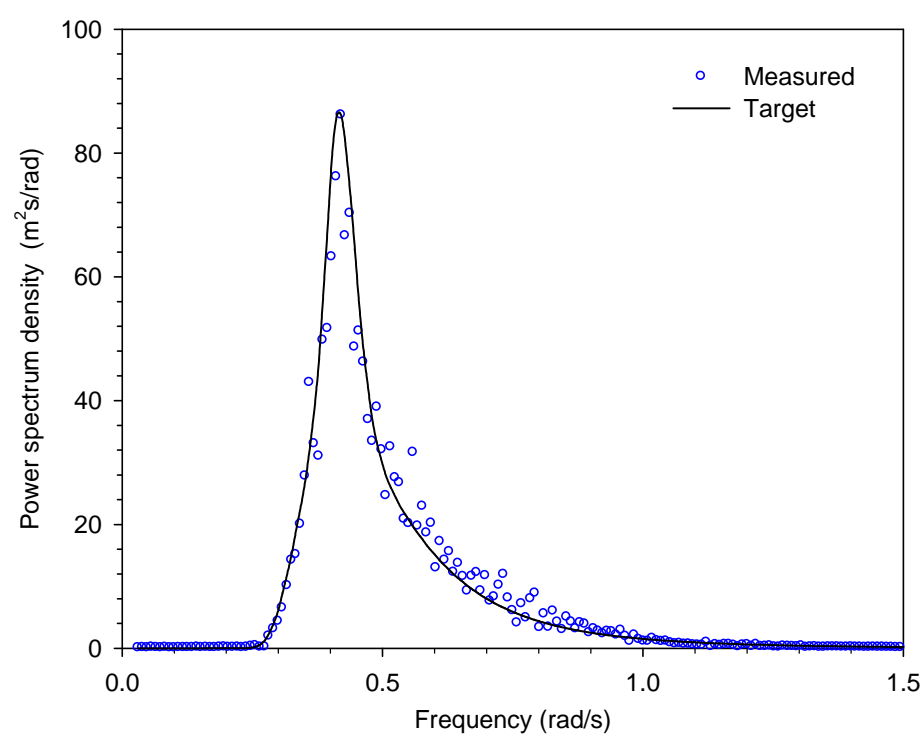

Fig. 3. Calibration result of wave spectrum 

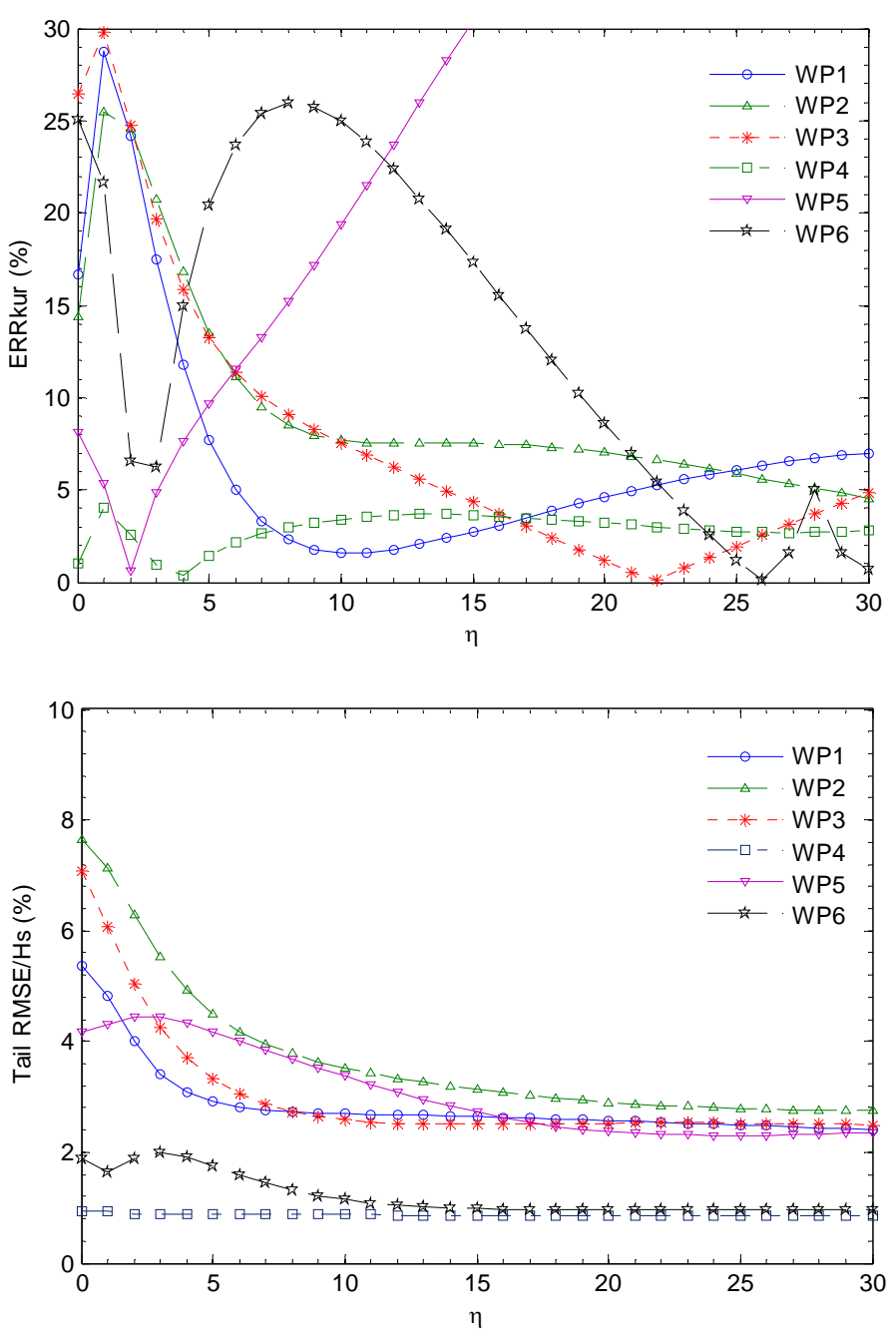

5

(a)

(b)

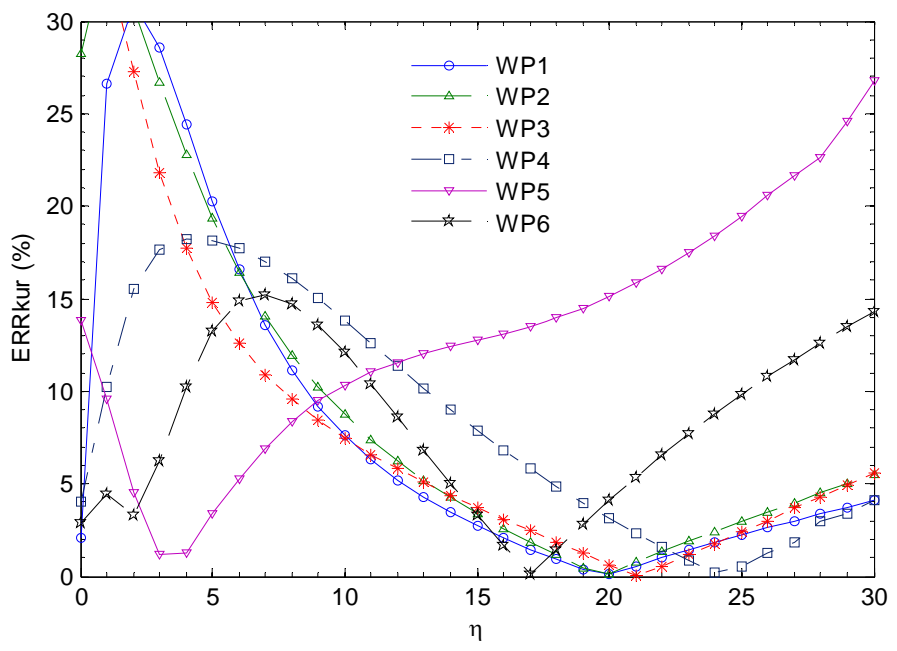




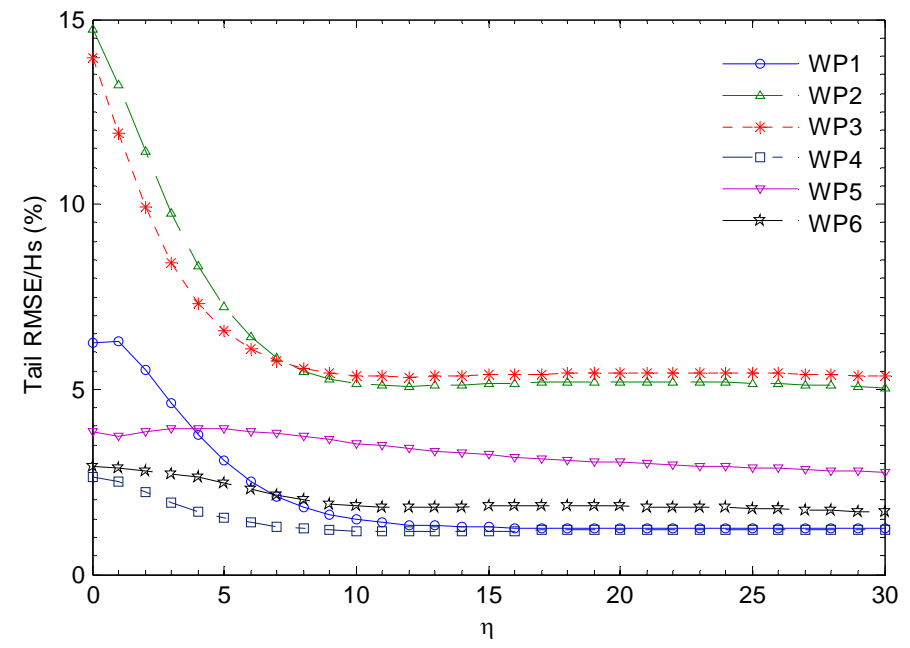

(c)

(d)
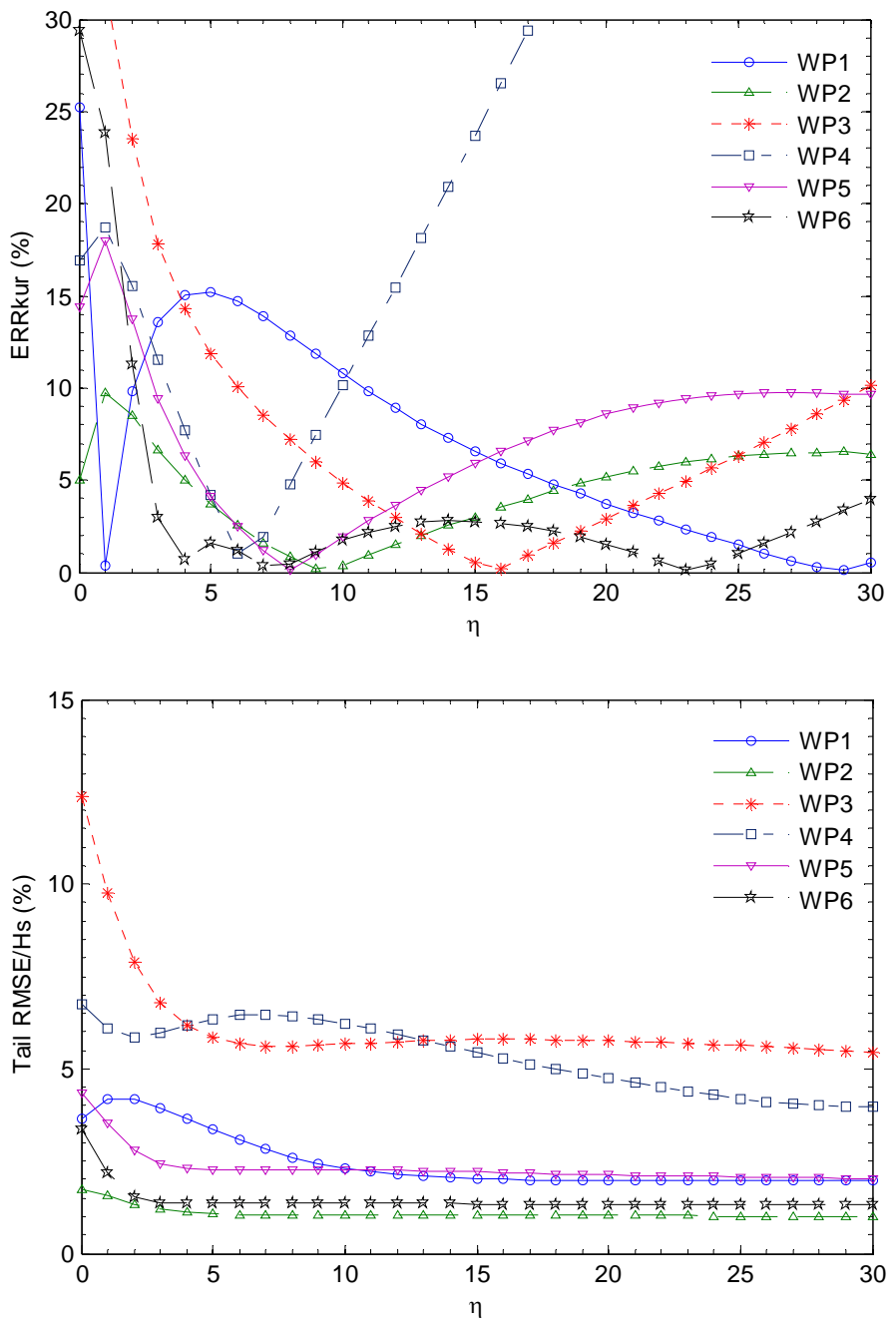

(e)

(f)

Fig. 4. Error of LH-kurtosis and tail RMSE of estimated quantiles at different levels: (a) Error of LH-kurtosis,

13 Head seas; (b) Tail RMSE, Head seas; (c) Error of LH-kurtosis, Quartering seas; (d) Tail RMSE, Quartering 14 seas; (e) Error of LH-kurtosis, Beam seas; (f) Tail RMSE, Beam seas 

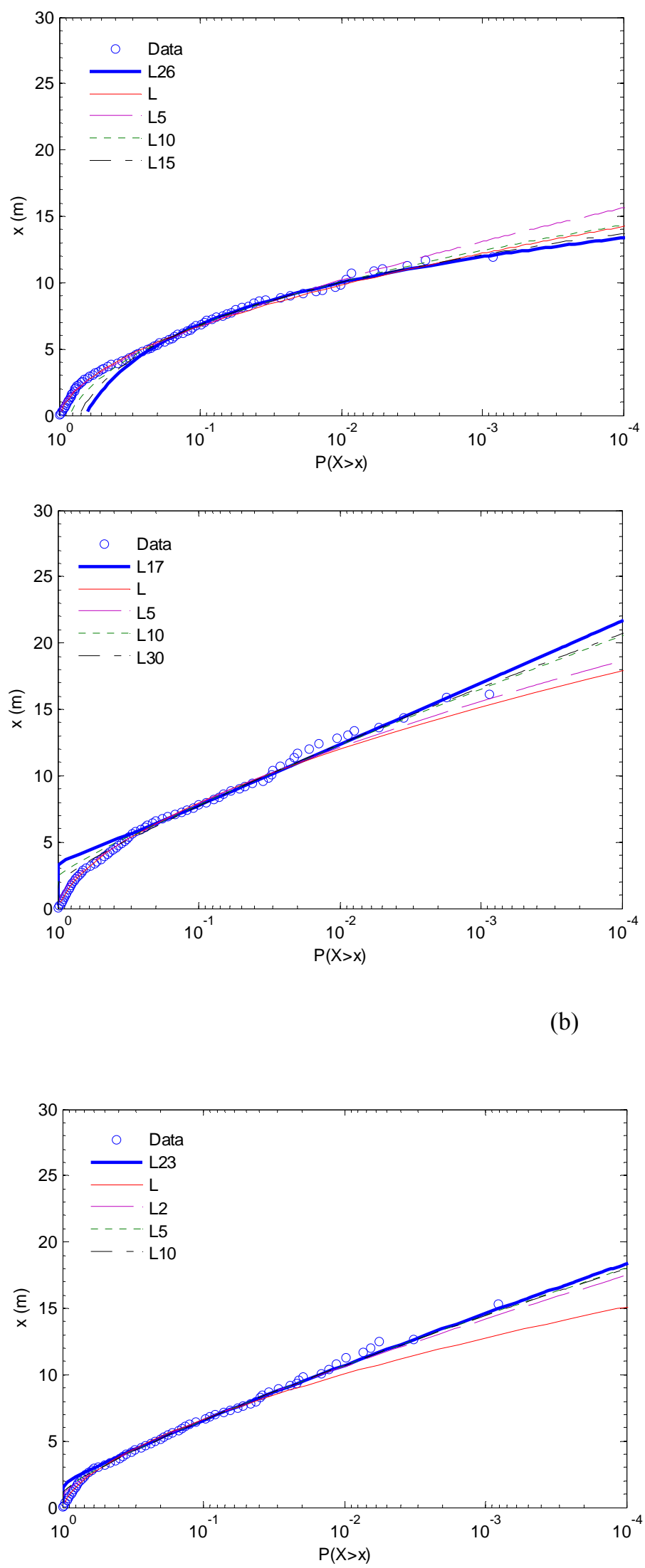


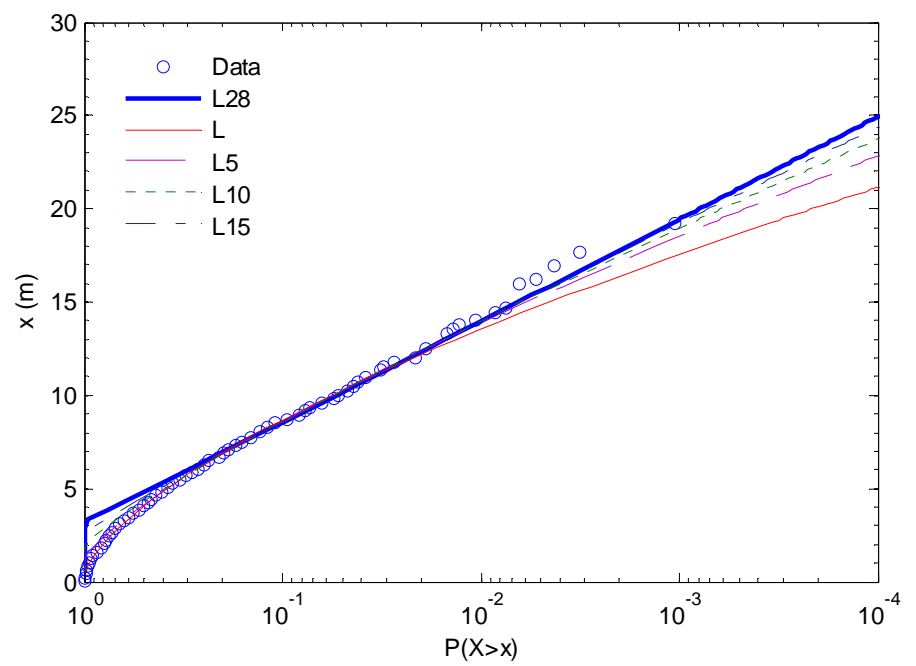

(c)

(d)

21 Fig. 5. Comparison of estimated exceedance probability distributions with measured data: (a) WP6, Head seas; (b) WP6, Quartering seas; (c) WP6, Beam seas; (d) Incident wave 

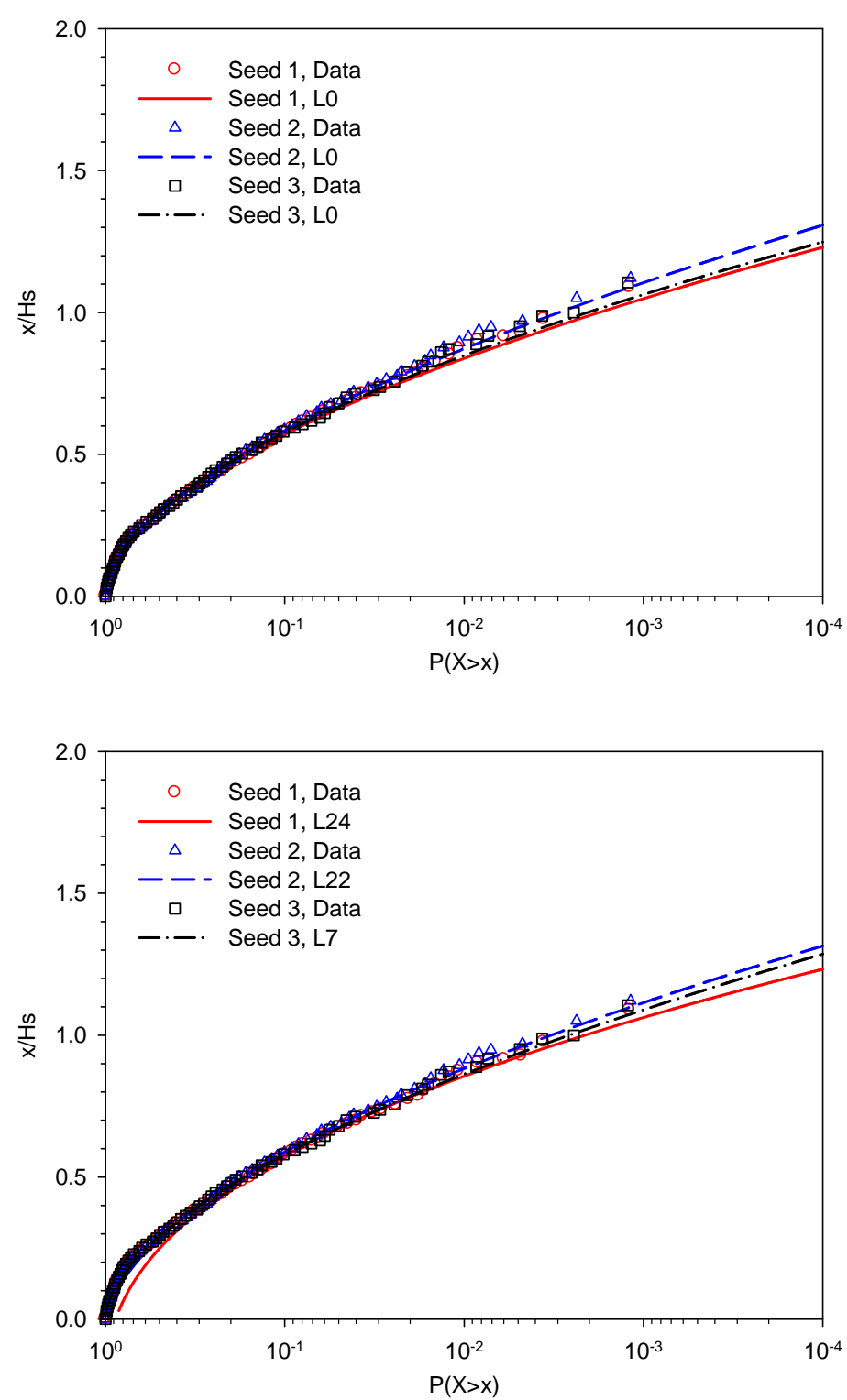

(b)

30 Fig. 6. Comparison of estimated exceedance probability distributions with measured data of wave crest 

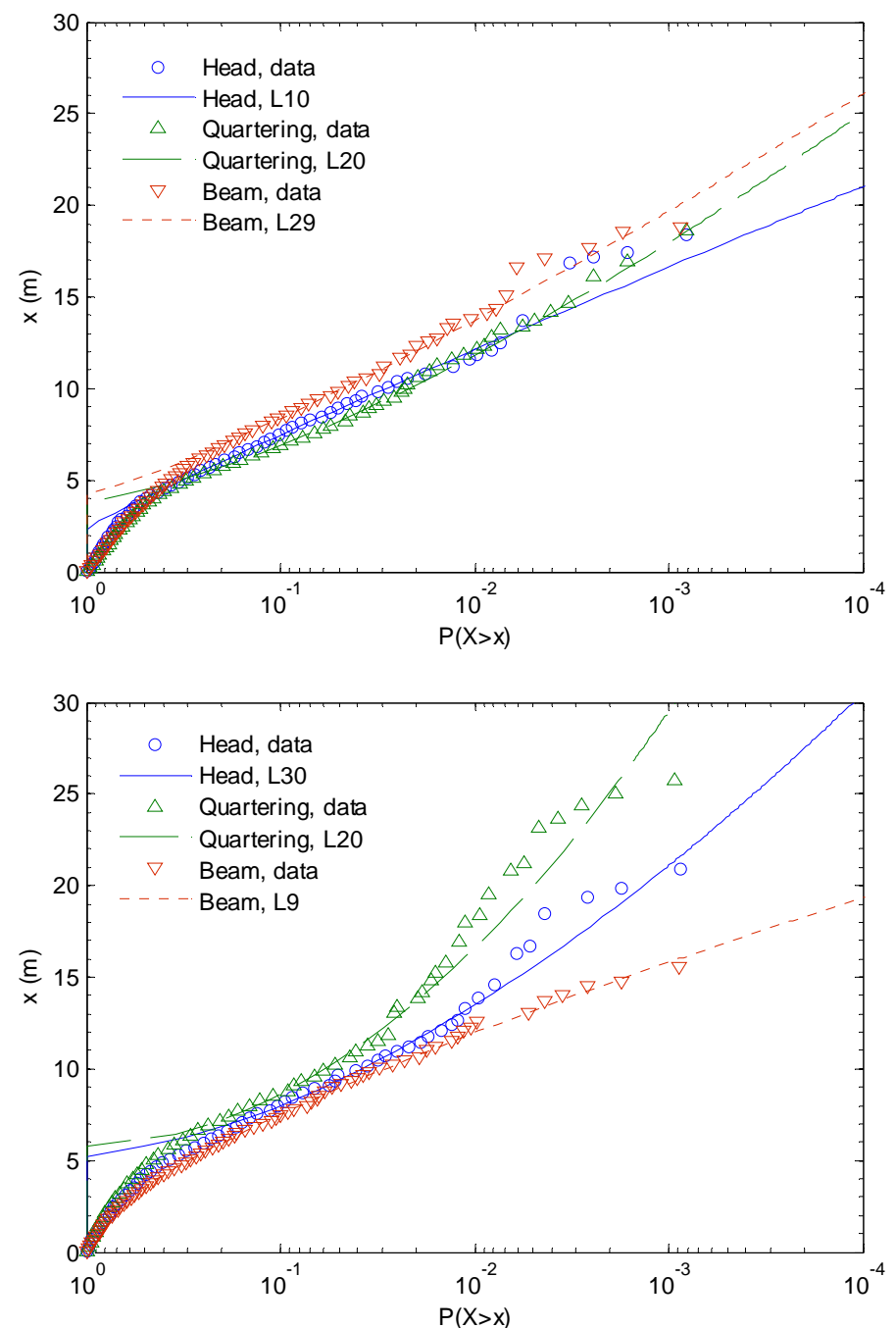

(a)

(b)

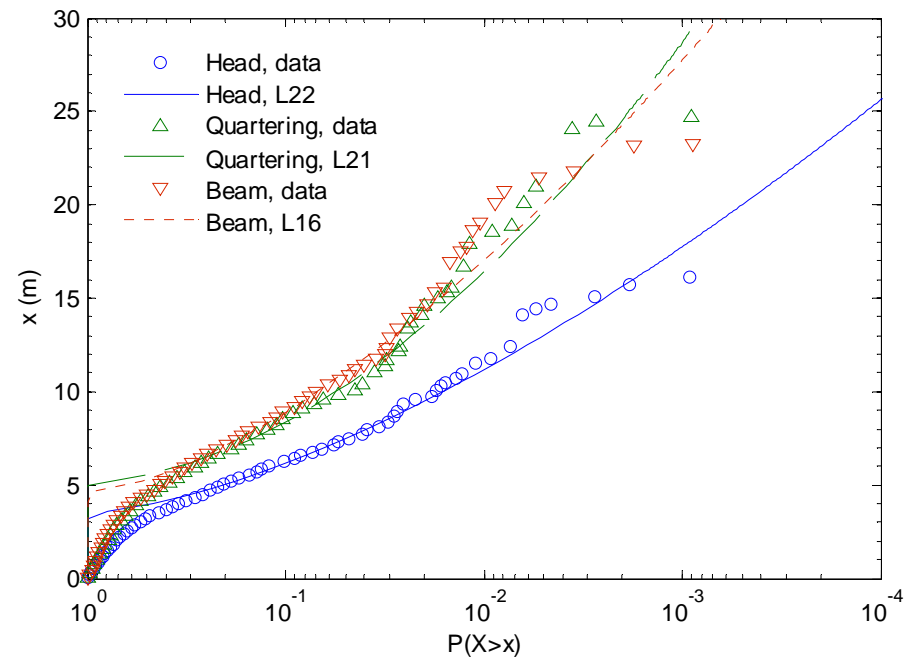




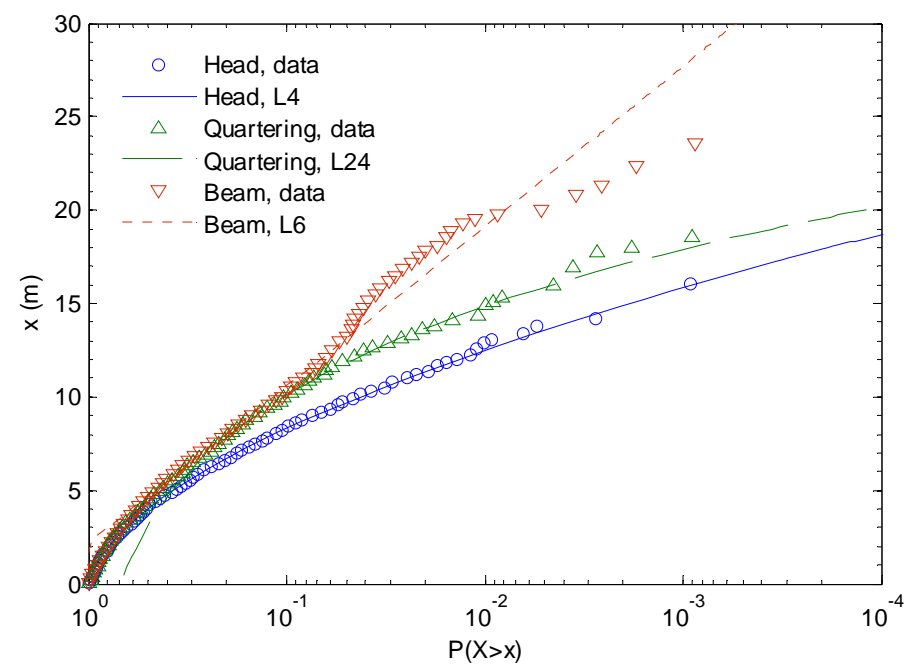

(c)

(d)
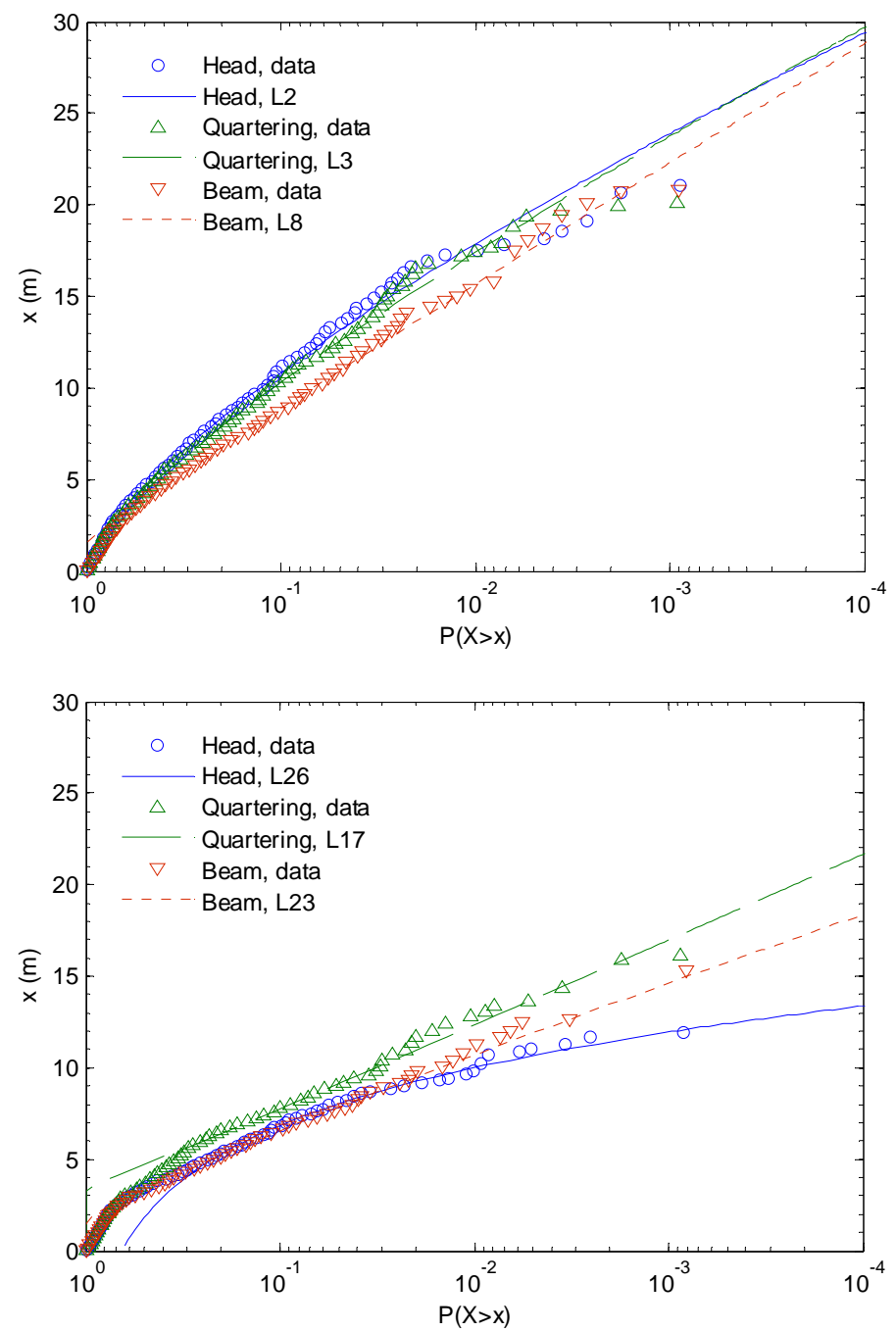

46 Fig. 7. Estimated exceedance probability distributions and measured data at different locations: (a) WP1; (b)

47 WP2; (c) WP3; (d) WP4; (e) WP5; (f) WP6 


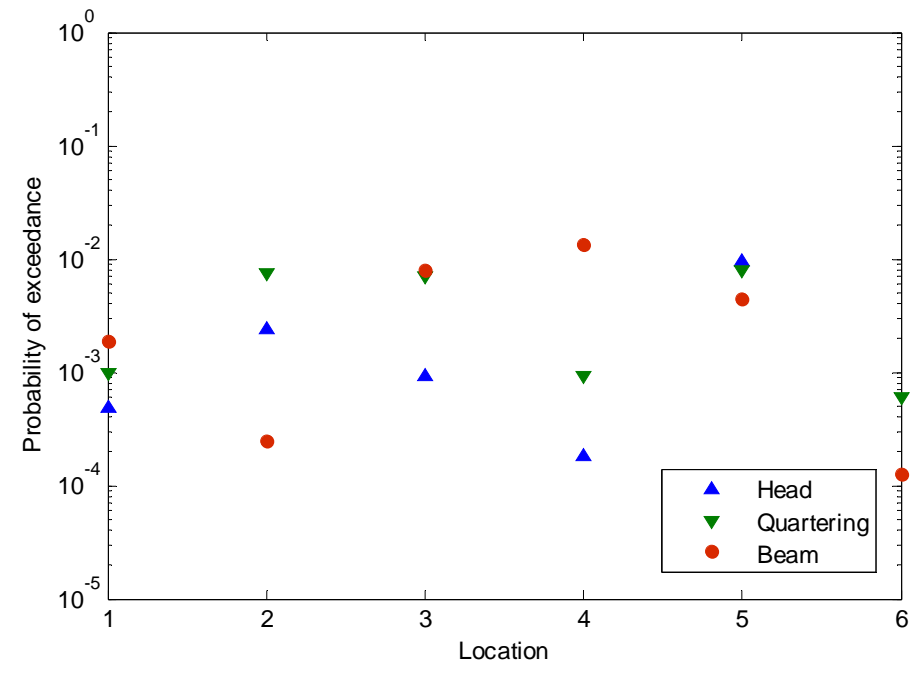

5Fig. 8. Estimated exceedance probabilities using LH-moments for still-water air gap of $18 \mathrm{~m}$

51 


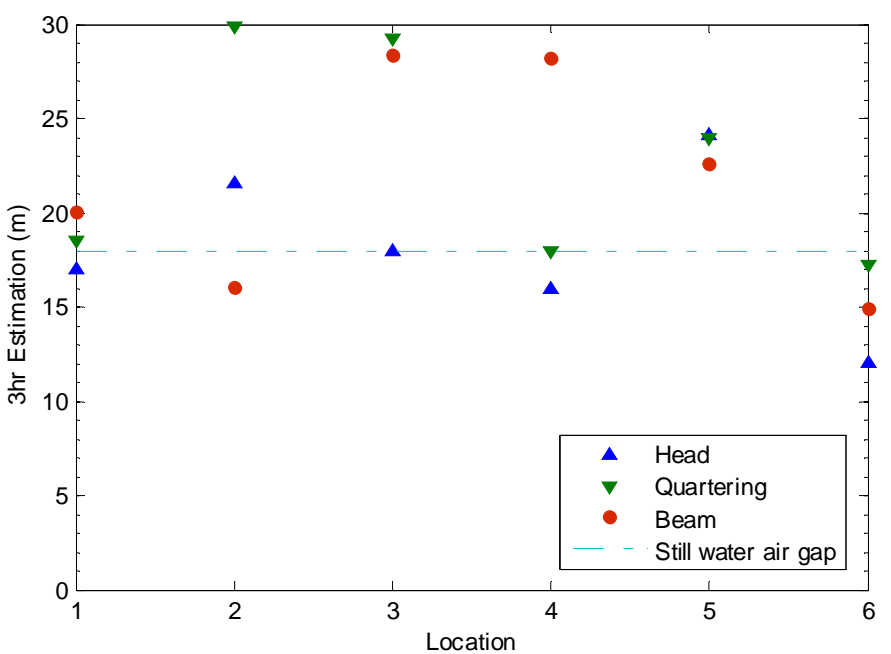

5Fig. 9. Estimated 3-h extremes using LH-moments compared to still-water air gap of $18 \mathrm{~m}$ 56

57 